\title{
Heavy Ion Physics with CMS at LHC
}

\author{
Pablo P. Yepes ${ }^{\text {a }}$ \\ for the CMS Collaboration

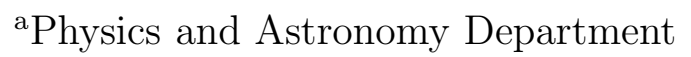 \\ MS 315, Rice University \\ Houston, TX 77005, USA \\ http://yepes.rice.edu \\ yepes@rice.edu
}

The CMS detector is well equipped to provide unique measurements in heavy ion collisions at LHC. It will provide measurements of the $\mathrm{J} / \Psi$ and $\Upsilon$ families with good separation of the different resonances. Jet quenching will be studied by analyzing monojet-to-dijet ratios and $Z^{0}(\gamma)+$ jet events.

\section{CMS Detector}

The Large Hadron Collider (LHC) plans to operate as a heavy ion collider for one month each year starting in 2007. LHC will be capable of accelerating a variety of ions up to beam energies of $7 \mathrm{TeV} /$ charge. The design, peak and three-hour-fill-averaged luminosities are presented in Table 1 along with the maximum center of mass energy [1]. The Compact Muon Solenoid (CMS) [2] experiment is a general-purpose facility to study

\begin{tabular}{|c|c|c|c|}
\hline Ion & $\begin{array}{c}L_{\max } \\
\left(\mathrm{cm}^{-2} \mathrm{~s}^{-1}\right)\end{array}$ & $\begin{array}{c}<\mathrm{L}> \\
\left(\mathrm{cm}^{-2} \mathrm{~s}^{-1}\right)\end{array}$ & $\begin{array}{c}\sqrt{s_{N N}} \\
(\mathrm{GeV})\end{array}$ \\
\hline${ }^{208} \mathrm{~Pb}^{82}$ & $1.010^{27}$ & $4.210^{26}$ & 5500 \\
${ }^{120} \mathrm{Sn}^{50}$ & $1.710^{28}$ & $7.610^{27}$ & 5800 \\
${ }^{84} \mathrm{Kr}^{36}$ & $6.610^{28}$ & $3.210^{28}$ & 6000 \\
${ }^{40} \mathrm{Ar}^{18}$ & $1.010^{30}$ & $5.210^{29}$ & 6300 \\
${ }^{16} \mathrm{O}^{8}$ & $3.110^{31}$ & $1.410^{31}$ & 7000 \\
\hline
\end{tabular}

Table 1

LHC planned ion beams with design, maximum and fill-averaged luminosities along with maximum center of mass energies.

hadronic collisions at the LHC. The detector consists of a tracking system, electromagnetic and hadronic calorimeters, and muon detectors. A solenoidal magnet providing a 4 Tesla magnetic field surrounds the tracking and calorimetric systems. The tracker, covering the rapidity region $|\eta|<2.5$, is based on silicon technology. The electromagnetic 
calorimeter consists of about 83000 lead-tungstate crystals arranged in a central barrel covering $|\eta|<1.48$ and the endcaps, which extend its range to rapidity $|\eta|<3$. In the central barrel, the granularity is as high as $\eta \mathrm{x} \phi=0.0175 \mathrm{x} 0.0175$. The hadronic calorimeter consists of barrel and endcap sections, each made of sandwiches of copper plates and plastic scintillator. In the central region $(|\eta|<2)$ the $\eta \mathrm{x} \phi$ segmentation is $0.087 \mathrm{x} 0.087$. The combination of electromagnetic and hadronic calorimeters provides coverage of the central rapidity region with excellent energy resolution. In addition, coverage at large rapidity $(3<|\eta|<5)$ is achieved by two very forward calorimeters. The large calorimetric coverage and good energy resolution makes CMS an optimal detector for jet studies.

The muon system covers the $|\eta|<2.5$ region. In the barrel $(|\eta|<1.5)$, muons must have a transverse momentum larger than $3.5 \mathrm{GeV} / c^{2}$ to be efficiently detected.

\section{Quarkonia Production}

The suppression of charmonium production has provided strong evidence for the formation of dense hot hadronic matter at SPS energies[3]. At RHIC, $J / \Psi$ production is an important part of the PHENIX [0] physics program. STAR is also expected to study charmonium production [5]. At LHC, CMS is well equipped to study bottonium and charmonium production. The ability to study $\Upsilon$ yields at LHC, which has a negligible cross sections at lower energies, will allow further understanding of the characteristics of dense hadronic matter.

A detailed study of the feasibility of quarkonia detection in CMS was carried out. Production cross sections were estimated utilizing the expression: $\sigma_{A A}=A^{2 \alpha} \sigma_{p p}$ with $\alpha=0.9$ (0.95) for $J / \Psi(\Upsilon)$. $\sigma_{p p}$ was obtained by extrapolating Tevatron data from $\sqrt{s}=1.8$ to 5.5(7) $\mathrm{TeV}$ for $\mathrm{PbPb}(\mathrm{CaCa})$. The obtained cross sections are shown in Table 2. The

\begin{tabular}{|c|r|r|r|r|r|r|}
\hline & \multicolumn{2}{|c|}{ Cross Sections } & \multicolumn{4}{|c|}{$\begin{array}{c}\text { Reconstructed } \\
\text { in 1 Month Run }\end{array}$} \\
\hline Ion & $\mathrm{PbPb}$ & CaCa & \multicolumn{2}{|c|}{$\mathrm{PbPb}$} & \multicolumn{2}{|c|}{ CaCa } \\
\hline & (mbarns $)$ & $(\mu$ barns $)$ & \# Events & $\mathrm{S} / \sqrt{B}$ & \# Events & $\mathrm{S} / \sqrt{B}$ \\
\hline$J / \Psi$ & 58. & 3600 & 10600 & 100 & 220000 & 1440 \\
$\Psi^{\prime}$ & 1.4 & 90 & 350 & 2.4 & 5800 & 92 \\
$\Upsilon$ & 0.4 & 21 & 22000 & 188 & 340000 & 1788 \\
$\Upsilon^{\prime}$ & 0.12 & 6.4 & 7500 & 64 & 115000 & 605 \\
$\Upsilon^{\prime \prime}$ & 0.04 & 2.1 & 2500 & 21 & 37734 & 198 \\
\hline
\end{tabular}

Table 2

Quarkonia cross sections at LHC along with the projected number of reconstructed events in CMS in the dimuon channel and the signal over the square root of the background.

muon system acceptance for quarkonia decaying into dimuons was studied using the barrel and the endcap detectors. In the barrel, only $J / \Psi$ particles with $p_{T}>5 \mathrm{GeV} / \mathrm{c}$ are detected, with an acceptance at the level of a few per cent. When the endcap chambers are included, $J / \Psi$ with $p_{T}=0$ is detected with an acceptance of $2 \%$. For $\Upsilon$, the acceptance 
is 30(15)\% when the end-cap detectors are (are not) considered, with little dependence on $p_{T}$. Quarkonia reconstruction was studied with detailed simulation and full reconstruction programs. Muon background from $\pi, \mathrm{K}$ and heavy quark leptonic decays was considered. The $p_{T}$ spectra for pions and kaons was obtained from SHAKER [6]. The average $p_{T}$, $0.48(0.67) \mathrm{GeV} / \mathrm{c}$ for pion(kaons), is higher than that obtained with other event generators. The multiplicity in central $\mathrm{PbPb}$ events is assumed to be $d N / d y=8000$. Given that our estimates are pessimistic, backgrounds are probably overestimated. Fig. 1 1 depicts the expected dimuon invariant mass spectrum in the $\Upsilon$ region for 1 month of $\mathrm{PbPb}$ running. Table 2 shows the number of expected reconstructed events for the $J / \Psi$ and $\Upsilon$ families

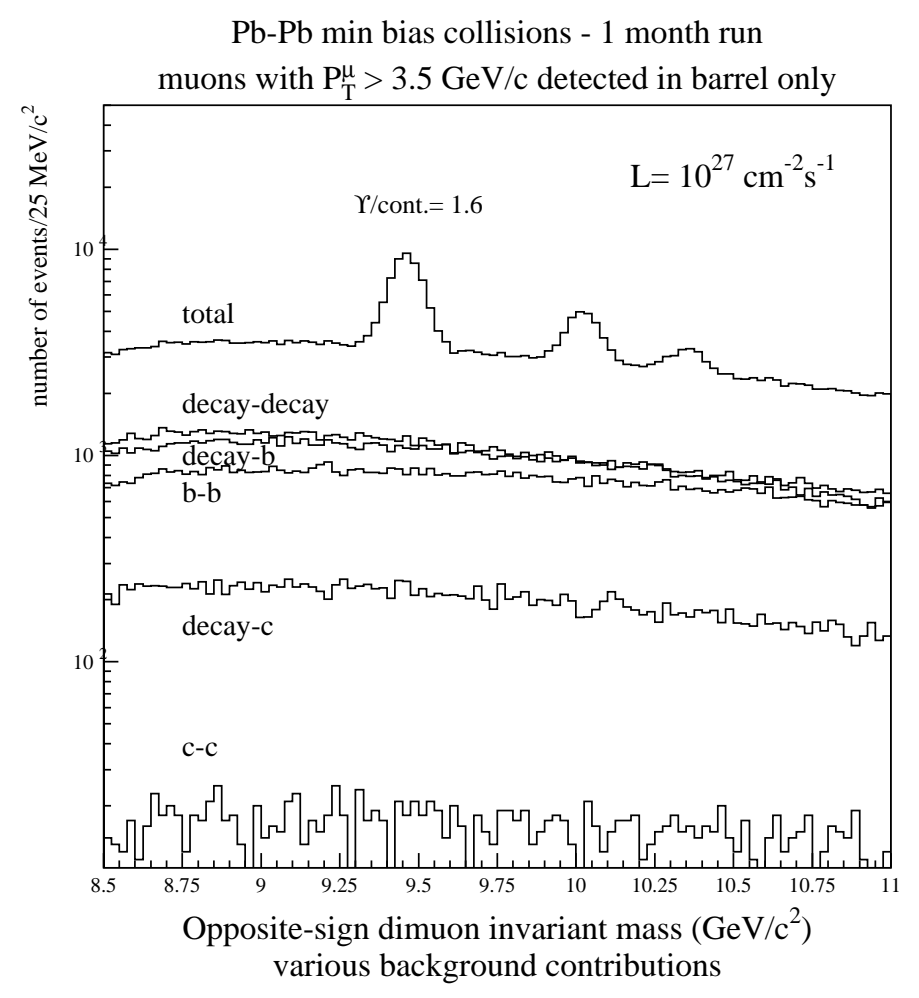

Figure 1. Invariant dimuon spectrum reconstructed with the barrel muon system expected in one month of running with $\mathrm{PbPb}$. The background from different sources is indicated along with the sum of all contributions.

with $\mathrm{PbPb}$ and with $\mathrm{CaCa}$ in one month running at design luminosity. In addition, the statistical significance of the signal, number of particles divided by the square root of the background, is also given.

\section{Jet Studies}

Recent results from RHIC [7, [] show a softening of the transverse momentum above 5 $\mathrm{GeV} / \mathrm{c}$ in central collisions. This could be attributed to jet quenching in the hot hadronic 
media produced after the collision. At LHC, $10^{7}$ dijets with $E_{T}^{\text {jet }}>100 \mathrm{GeV}$ are produced in the region $|\eta|<2.6$ in a one-month run of $\mathrm{PbPb}$. The number is reduced by about a factor of 2 if only the barrel is considered, $|\eta|<1$.5. At LHC, jet reconstruction in $\mathrm{PbPb}$ is possible, even for central collisions. A modified version of the UA1 jet-finding algorithm was used to reconstruct jets in central $\mathrm{PbPb}$ collisions using the information provided by the electromagnetic and hadronic calorimeters. As in the quarkonia study, the multiplicity in central $\mathrm{PbPb}$ events is assumed to be $d N / d y=8000$. Table 3 shows the efficiency,

\begin{tabular}{|c|c|c|c|}
\hline$E_{T}^{\min }(\mathrm{GeV})$ & Efficiency $(\%)$ & Contamination $(\%)$ & $\sigma\left(E_{T}\right) / E_{T}(\%)$ \\
\hline 50 & $94 \pm 3$ & $12.0 \pm 3$ & 16.7 \\
100 & $100 \pm 2$ & $1.0 \pm 0.4$ & 11.6 \\
150 & $98 \pm 2$ & $0.4 \pm 0.3$ & 9.2 \\
200 & $99 \pm 2$ & $0.4 \pm 0.3$ & 8.6 \\
\hline
\end{tabular}

Table 3

Efficiency, contamination, and energy resolution for jets above different transverse momentum thresholds in central $\mathrm{PbPb}$ collisions.

contamination and energy resolution for finding jets above certain thresholds. As can be seen, even the lowest threshold jets can be reconstructed with good efficiency and relatively low background. This good performance will allow CMS to study jet quenching in dijet production [8]. Jet energy loss in hadronic matter can also be measured by tagging jets opposite to non-strongly interacting particles such as $Z^{0}$ or gamma, both produced in the reactions $q g \rightarrow q V$ and $q \bar{q} \rightarrow g V$, where $\mathrm{V}=Z^{0}$ or $\gamma$.

\section{Conclusions}

In conclusion, CMS provides a unique tool to study heavy ion collisions at the LHC. It will probe hot hadronic matter by studying $\mathrm{J} / \Psi$ and $\Upsilon$ production. Its excellent calorimetry will provide large coverage and good energy resolution for jet quenching studies.

\section{REFERENCES}

1. D. Brandt, $5^{\text {th }}$ CMS Heavy Ion Meeting, St. Petersburg, June 11-14, 2000.

2. The Compact Muon Solenoid, Technical Proposal, CERN/LHCC 94-38, LHCC/P1, Dec 15, 1994.

3. M. C. Abreu et al. [NA50 Collaboration], Phys. Lett. B 477, 28 (2000).

4. W. Zajc, Recent Results from PHENIX, in these proceedings.

5. T. J. LeCompte [STAR Collaboration], Workshop on Quarkonium Production in Relativistic Nuclear Collisions, Seattle, WA, 11-15 May 1998..

6. F. Antinori, CERN/ALICE 93-01 (MC).

7. J. Harris, Recent Results from STAR, in these proceedings.

8. M. Gyulassy and M. Plumer, Phys. Lett. B 243, 432 (1990). 